\title{
Blood-filled cerebrospinal fluid-enhanced pericyte microvasculature contraction in rat retina: A novel in vitro study of subarachnoid hemorrhage
}

\author{
ZHI LIU ${ }^{1}$, QIANG LI ${ }^{1}$, GAOYU CUI ${ }^{1}$, GANG ZHU ${ }^{1}$, WEIHUA TANG ${ }^{1}$, \\ HENGLI ZHAO ${ }^{1}$, JOHN H. ZHANG ${ }^{2}$, YUJIE $\mathrm{CHEN}^{1}$ and HUA FENG ${ }^{1}$ \\ ${ }^{1}$ Department of Neurosurgery, Southwest Hospital, Third Military Medical University, Chongqing 400038, P.R. China; \\ ${ }^{2}$ Department of Anesthesiology, Neurosurgery and Physiology, Loma Linda University, Loma Linda, CA 92350, USA
}

Received December 21, 2015; Accepted February 10, 2016

DOI: $10.3892 /$ etm.2016.3644

\begin{abstract}
Previously, it was widely accepted that the delayed ischemic injury and poor clinical outcome following subarachnoid hemorrhage (SAH) was caused by cerebral vasospasm. This classical theory was challenged by a clazosentan clinical trial, which failed to improve patient outcome, despite reversing angiographic vasospasm. One possible explanation for the results of this trial is the changes in microcirculation following $\mathrm{SAH}$, particularly in pericytes, which are the primary cell type controlling microcirculation in the brain parenchyma. However, as a result of technical limitations and the lack of suitable models, there was no direct evidence of microvessel dysfunction following SAH. In the present study, whole-mount retinal microvasculature has been introduced to study microcirculation in the brain following experimental $\mathrm{SAH}$ in vitro. Artificial blood-filled cerebrospinal fluid (BSCF) was applied to the retinal microvasculature to test the hypothesis that the presence of subarachnoid blood affects the contractile properties of the pericytes containing cerebral microcirculation during the early phase of SAH. It was observed that BCSF induced retina microvessel contraction and that this contraction could be resolved by BCSF wash-out. Furthermore, BCSF application accelerated pericyte-populated collagen gel contraction and increased the expression of $\alpha$-smooth muscle actin. In addition, BCSF induced an influx of calcium in cultured retinal pericytes. In conclusion, the present study demonstrates increased contractility of retinal microvessels and pericytes in the presence of BCSF in vitro. These findings
\end{abstract}

Correspondence to: Dr Hua Feng or Dr Yujie Chen, Department of Neurosurgery, Southwest Hospital, Third Military Medical University, 29 Gaotanyan Street, Shapingba, Chongqing 400038, P.R. China

E-mail: fenghua8888@vip.163.com

E-mail: yujiechen6886@foxmail.com

Key words: subarachnoid hemorrhage, microcirculation, blood-filled cerebrospinal fluid, retinal microvessel, pericyte suggest that pericyte contraction and microvascular dysfunction is induced following SAH, which could lead to greater susceptibility to SAH-induced ischemia.

\section{Introduction}

Despite years of intensive clinical and experimental investigation, subarachnoid hemorrhage $(\mathrm{SAH})$ remains a lethal complication of a ruptured intracranial aneurysm. For survivors of the initial bleeding, delayed cerebral ischemia frequently occurs and is the primary cause of subsequent morbidity and mortality (1). However, the mechanism of delayed cerebral ischemia following SAH is poorly understood (2). Previous studies have typically focused on vasospasm of the large cerebral arteries; however, a double-blind, randomized clinical trial of the endothelin receptor antagonist clazosentan showed no effect on clinical outcome, despite inhibiting angiographic vasospasm (3).

An explanation for these results could be that cerebral microcirculation and its regulatory mechanisms are directly affected by SAH (4), particularly pericytes, the primary cell type controlling microcirculation in brain parenchyma (5). A number of studies have addressed the role of microcirculatory dysfunction during $\mathrm{SAH}$, where arteriolar constriction is typically observed (6,7). However, these results and insights have not been confirmed in vitro. Until recently, in vitro observation and quantitative functional assessment of cerebral microcirculation following SAH were limited by the absence of suitable models.

The present study introduces whole-mount retinal microvasculature to study brain microcirculation following experimental SAH in vitro. Artificial blood-filled cerebrospinal fluid (BSCF) was applied to the mount to test the hypothesis that the presence of subarachnoid blood affects the contractile properties of pericytes containing cerebral microcirculation during the early phase of SAH.

\section{Materials and methods}

Experimental animals. All protocols used were approved by the Ethics Committee of the Southwest Hospital (Chongqing, 
China) and performed in accordance with the guidelines of the eighth edition of the National Institutes of Health Guide for the Care and Use of Laboratory Animals (8). Sixty-five six-week old male Sprague-Dawley rats (Experimental Animal Center, Third Military Medical University, Chongqing, China), weighing between 200 and $250 \mathrm{~g}$, were used in the present study. All rats were kept in a quiet room at $23-25^{\circ} \mathrm{C}, 70 \%$ humidity and a $12 \mathrm{~h}$ light/dark cycle and were allowed $\mathrm{ad}$ libitum access to normal rat chow and filtered water.

BCSF preparation. BCSF was prepared as previously described (9). Briefly, equal volumes of arterial blood and normal cerebrospinal fluid from donor rats was mixed and incubated in a $37^{\circ} \mathrm{C}$ water bath for $24 \mathrm{~h}$. Then, the mixed samples were centrifuged at $10,000 \mathrm{x} \mathrm{g}$ for $20 \mathrm{~min}$ at room temperature. Finally, the supernatant was collected and stored at $4^{\circ} \mathrm{C}$ until use. BCSF was freshly prepared under aseptic conditions prior to the experiments.

Whole-mount retina preparation. Whole-mount retina was prepared as previously described (10). Briefly, rats were anaesthetized through intraperitoneal injection of ketamine (50 mg/kg, Gutian Pharmaceutical Co., Ltd., Fujian, China) and xylazine (10 mg/kg, Sigma-Aldrich, Shanghai, China) prior to sacrifice by decapitation. Following sacrifice, the eyes were immediately enucleated. A small cut was then made in the sclera, close to the cornea, and the eyeball was submerged in Ames' medium (Sigma-Aldrich) equilibrated with $95 \% \mathrm{O}_{2}$ and $5 \% \mathrm{CO}_{2}$. Then, the retina were carefully dissected from the pigment epithelium and attached, ganglion cell side-up, to a MF-Millipore Membrane Filter (EMD Millipore, Billerica, MA, USA; cat. no. AABP02500) with a $2 \mathrm{~mm}$ diameter hole in the center for microvascular observation during imaging.

Time-lapse photography. The whole-mount retina preparation was transferred into a $0.5 \mathrm{ml}$ imaging chamber on the fixed stage of an upright microscope (Leica DM LFSA; Leica Microsystems GmbH, Wetzlar, Germany). The preparation was continuously superfused with oxygenated bicarbonate-buffered Ames' medium at $35^{\circ} \mathrm{C}$. Microvessels were viewed at x400 magnification with the aid of a $\mathrm{x} 40$ water-immersion objective. Following a $2.67 \mathrm{~min}$ control period, microvessels were exposed to the experimental perfusate for $5.33 \mathrm{~min}$, then re-exposed to the control perfusate. To facilitate the detection of pericyte contractions, time-lapse images were captured at $8 \mathrm{sec}$ intervals using a digital camera running Image-Pro Plus software version 6.0 (Media Cybernetics, Inc., Rockville, MD, USA). The small number of pericytes $(<5 \%)$ that spontaneously contracted and relaxed were excluded from analysis. Based on the knowledge that $20 \%$ of the length of microvessels of the rat retina are within $30 \mu \mathrm{m}$ of a bifurcation (11), the probability of responding pericytes being located near microvessel branch points $(\leq 30 \mu \mathrm{m})$ was calculated as previously described (12). Lumen diameters at sites adjacent to contracting pericytes were measured using Image-Pro Plus software. During exposure to experimental perfusates, lumen diameters were measured at the time of maximum change in responsive vessels. As contracting pericytes can cause microvascular lumens to move out of the narrow depth of focus, only lumens that remained in focus throughout the experiment were included in the analysis.
In vitro culture of retinal pericytes. Microvessel pericytes were obtained from rat retina as previously described (13). Rat retinas were dissected as described above. The tissue was then washed in a phosphate-buffered saline (PBS) solution supplemented with antibiotics $(100 \mathrm{U} / \mathrm{ml}$ penicillin and $100 \mathrm{U} / \mathrm{ml}$ streptomycin), then minced and incubated in PBS solution with $0.1 \%$ collagenase, $0.2 \%$ trypsin and $0.02 \%$ glucose (all purchased from Sigma-Aldrich). Next, the solution was homogenized for $\sim 60 \mathrm{~min}$ at $37^{\circ} \mathrm{C}$ on a shaking platform. The suspension was then filtered through $100 \mu$ m nylon mesh to remove large tissue fragments and washed in Dulbecco's modified Eagle's medium (DMEM, Hyclone; GE Healthcare Life Sciences, Logan, UT, USA) containing 10\% fetal bovine serum (Hyclone; GE Healthcare Life Sciences) and antibiotic solution (100 U/ml penicillin and $100 \mu \mathrm{g} / \mathrm{ml}$ streptomycin). Each culture cell dish was seeded from 4 retinas. Pure cultures of pericytes were incubated at $37^{\circ} \mathrm{C}$ with $5 \% \mathrm{CO}_{2}$ atmosphere, DMEM with $20 \%$ fetal bovine serum was changed daily (days 3, 4, and 5) until day 5, after which the medium was changed every 3 days. When primary pericyte cultures were near confluence $(>80 \%)$, they were further propagated by treatment with $1 \mathrm{ml}$ of $0.25 \%$ (Santa Cruz Biotechnology, Inc., Santa Cruz, CA, USA) and 0.02\% EDTA for $3 \mathrm{~min}$ and then were split into a 1:3 ratios to passage to 6-well plates. In the present study, cultured pericytes were identified using $\alpha$-smooth muscle actin ( $\alpha$-SMA) as a marker and morphological images were identified after the 3rd passage, and used between the 5th and 9th passages.

Collagen gel contraction assay. The collagen gel contraction assay was performed as described previously (14). Rat-tail tendon type I collagen (Shengyou Biotechnology Co., Ltd., Hangzhou, China) was diluted with DMEM to $2 \mathrm{mg} / \mathrm{ml}$ and adjusted to $\mathrm{pH}$ 7.4. To promote gel detachment, 12-well plates were precoated with $1 \%$ agarose. Retinal pericyte cultures were trypsinized when $80 \%$ confluent and liberated cells were counted using a hemocytometer. The trypsinized solution was then diluted to $1 \times 10^{5}$ cells/ml with DMEM. Equal volumes of collagen and cell solution were then combined, and $1 \mathrm{ml}$ containing $1 \mathrm{mg}$ collagen and $5 \times 10^{4}$ cells was pipetted into each well. Then, gels were polymerized at $37^{\circ} \mathrm{C}$ and then released from the dish edges with a fine needle. Diameters were recorded at three time points: Prior to BCSF incubation, and BCSF incubation at 24 and $48 \mathrm{~h}$, with contraction measured relative to the initial gel diameter. All assays were repeated $3 \mathrm{x}$ with triplicate wells for each experimental condition.

Western blotting. Pericytes harvested from the collagen gel were suspended in ice-cold PBS at $1.25 \times 10^{6}$ cells $/ \mathrm{ml}$. Proteins were extracted by Total Protein Extraction Kit for Cultured Cells (Boster Biological Technology, Wuhan, China), following the instruction manual. Western blot analysis was then performed as previously described (15). Equivalent protein amounts $(30 \mu \mathrm{g})$ were loaded in each lane of 10\% SDS-PAGE gels. After gel electrophoresis, protein was transferred onto a nitrocellulose membrane, which was then blocked by $5 \%$ non-fat milk blocking buffer for $2 \mathrm{~h}$ at room temperature. The following primary antibodies were diluted to incubate with the membrane under gentle agitation at $4^{\circ} \mathrm{C}$ overnight: Anti- $\alpha$-SMA (1:1,000; cat. no. ab32575; rabbit monoclonal; Abcam, Cambridge, UK). Then, a secondary antibody (horseradish peroxidase-conjugated goat 
A

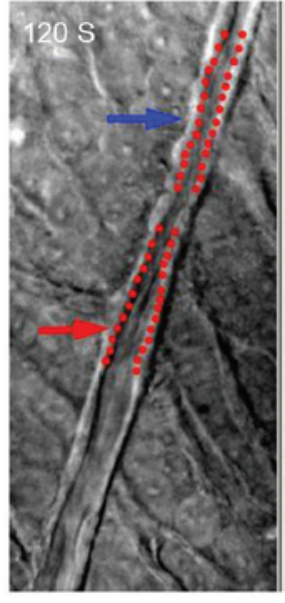

B

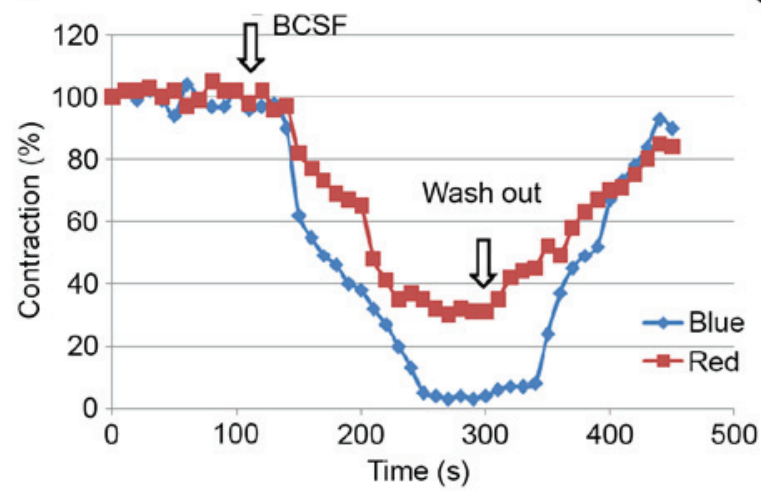

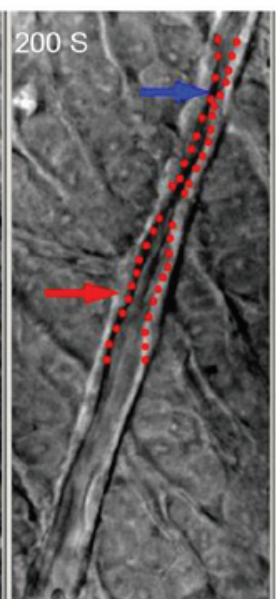

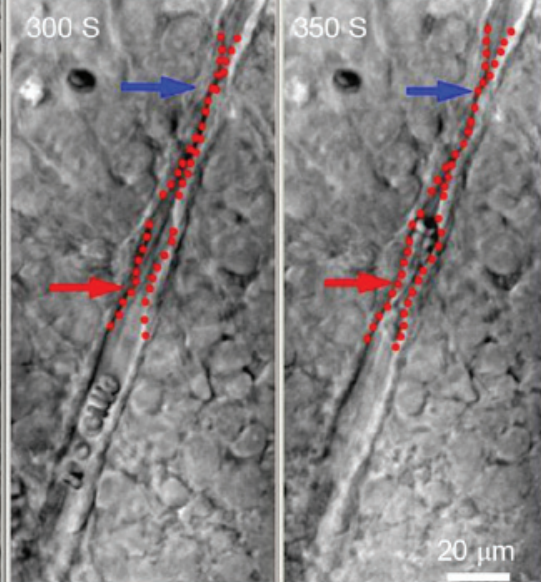

$\mathrm{C}$

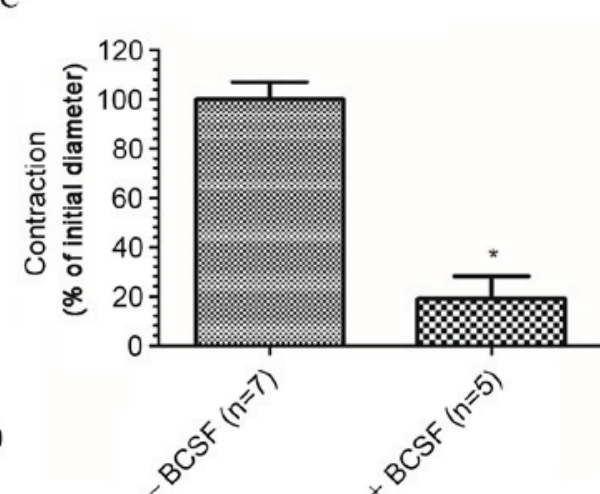

Figure 1. BCSF-induced contraction of pericyte-containing microvessels. (A) Representative time-lapse photographs of a retinal microvessel following BCSF exposure. Following BCSF exposure, microvessels contracted at the bifurcational point (red arrows) and the straight point (blue arrows). The right section of this panel is a sketch map, which illustrates the pericyte (light blue) and microvessels (red). (B) Time course of microvessel contraction. Each point indicates the percentage of the microvessel diameter relative to the original. (C) Quantitative analysis of the mean constriction in the control and BCSF-exposed group. "P<0.01 vs. -BCSF control. S, sec; BCSF, blood-filled cerebrospinal fluid.

anti-rabbit IgG; 1:1,000; cat. no. ab6721; Abcam) was incubated with the nitrocellulose membrane for $2 \mathrm{~h}$ at room temperature. Chemiluminescent detection was performed to identify the immune bands with an ELC Plus kit (cat. no. PRN2232; GE Healthcare Life Sciences). The resulting blots were scanned and semi-quantitatively analyzed in a blind fashion using Image $\mathrm{J}$ software version 1.48 (https://imagej.nih.gov/ij/). Anti- $\beta$-tubulin (1:4,000; Santa Cruz Biotechnology, Inc., Santa Cruz, CA) was used as an internal control for all the experiments and any changes observed were expressed as a percentage of the readings on day 0 .

Intracellular calcium $\left(\left[\mathrm{Ca}^{2+}\right]_{i}\right)$ microfluorimetry. $\left[\mathrm{Ca}^{2+}\right]_{\mathrm{i}}$ imaging was performed as previously described (16). The buffer solution for $\left[\mathrm{Ca}^{2+}\right]_{\mathrm{i}}$ measurement was $\mathrm{pH}$ adjusted to 7.4 with $\mathrm{NaOH}$ and contained (in mmol/l): $145 \mathrm{NaCl}, 1 \mathrm{CaCl}_{2}, 3 \mathrm{KCl}, 1 \mathrm{MgCl}_{2}, 10$ HEPES and 10 glucose. EGTA $(0.1 \mathrm{mmol} / \mathrm{l})$ was included in the $\mathrm{Ca}^{2+}$-free extracellular buffer. Cells were loaded into the microfluorimeter with the $\mathrm{Ca}^{2+}$-specific fluorescence indicator fura-2-acetoxymethylester $(3 \mathrm{mmol} / \mathrm{l})$ and incubated for $30 \mathrm{~min}$ at room temperature in the buffer solution. After loading, coverslips were placed on the bottom of a $600 \mathrm{ml}$ Plexiglas perfusion chamber with openings at either end for perfusion and aspiration. The cells were perfused with the buffer solution for $10 \mathrm{~min}$ prior to the experiment to allow de-esterification of the dye. Digital $\left[\mathrm{Ca}^{2+}\right]_{\mathrm{i}}$ imaging was performed through video microfluorimetry using a charge-coupled device (Princeton Instruments, Inc., Trenton, NJ, USA) attached to a Nikon Eclipse microscope with a Nikon CFI Super Fluor Objectives (Nikon Corporation, Tokyo, Japan) and MetaMorph System version 5.0 (Universal Imaging Corp, Downingtown, PA, USA). Imaging was performed with alternating excitation wavelengths of 340 and $380 \mathrm{~nm}$. Background fluorescence obtained from a cell-free portion of the same coverslip was subtracted from all recordings to normalize the data prior to calculation of the ratio between the 340:380 $\mathrm{nm}$ recordings. The ratio values were then converted into $\left[\mathrm{Ca}^{2+}\right]_{\mathrm{i}}$ as previously described (16).

Statistical analysis. The results are expressed as the mean \pm standard error of the mean. The statistical significance of the differences between groups was calculated using one-way analysis of variance followed by a Newman-Keuls test. $\mathrm{P}<0.05$ was considered to indicate a statistically significant difference. All statistical analyses were performed using SPSS software (version 16.0; SPSS, Inc., Chicago, IL, USA).

\section{Results}

BCSF induced contraction of retinal microvessels. To test the hypothesis that SAH would affect the physiology of retinal 
A

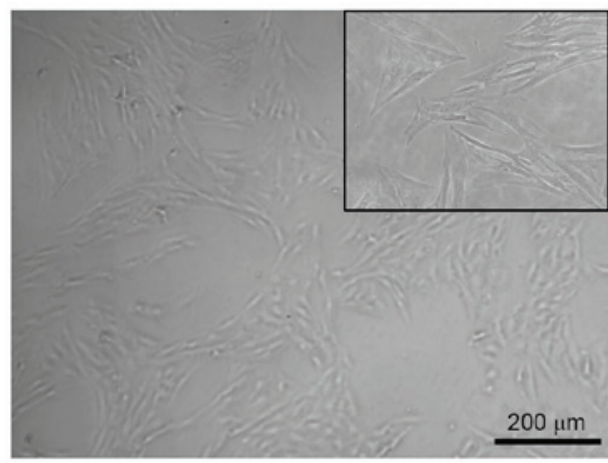

$\mathrm{C}$

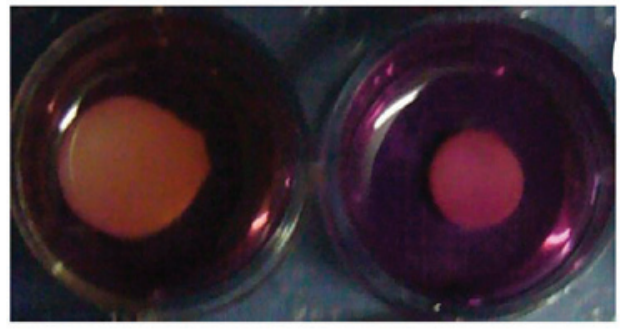

Saline

$\mathrm{E}$

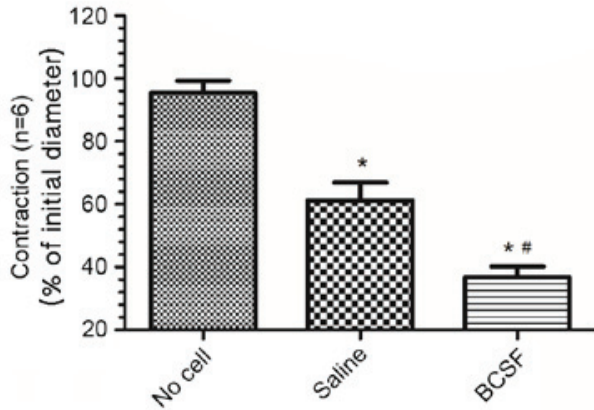

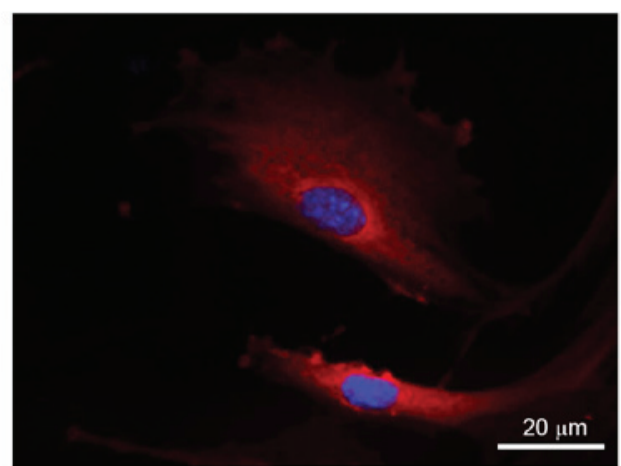

$\alpha-S M A / D A P I$

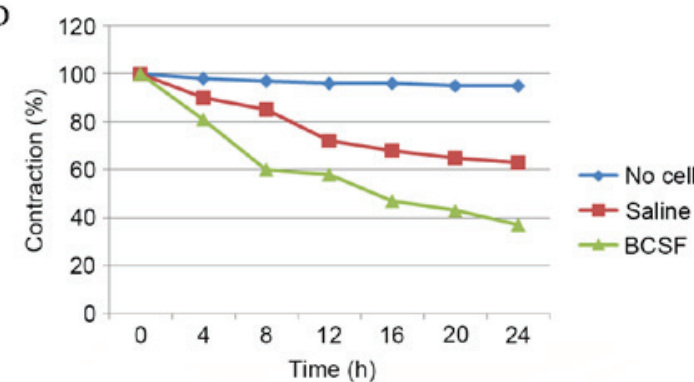

$\mathrm{F}$
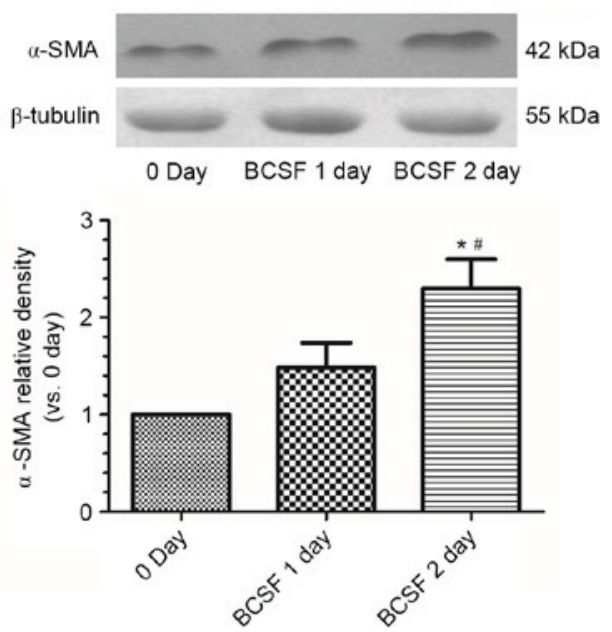

Figure 2. BCSF-induced pericyte-populated collagen gel contraction. (A) Representative morphological image of cultured retinal pericytes. (B) Immunofluorescence staining for $\alpha$-SMA on cultured retinal pericytes. (C) Representative image of pericyte-populated gels without BCSF (saline) and with BCSF exposure after $24 \mathrm{~h}$, showing BCSF accelerated gel contraction. (D) Quantitative analysis of the time course of the pericyte-populated collagen gel contraction in each group. (E) Quantitative analysis of the mean constriction of gel contraction in each group, showing that BCSF enhanced pericyte-populated gel contraction. (F) Representative western blot and quantitative analysis of $\alpha$-SMA expression in cultured retinal pericytes after 1 and 2 days of BCSF incubation, showing that BCSF increased the expression of $\alpha$-SMA in cultured pericytes. Each group, $n=6$. ${ }^{\prime \prime} \mathrm{P}<0.05$ vs. no cell or without BCSF groups. ${ }^{\prime \prime} \mathrm{P}<0.05$ vs. Cells + saline or BCSF 1 day groups. $\alpha$-SMA, alpha-smooth muscle actin; DAPI, 4',6-diamidino-2-phenylindole; BCSF, blood-filled cerebrospinal fluid.

microvessels, BSCF was used to mimic SAH in vitro. It was observed that exposure of retinal microvessels to BCSF caused increased contraction of the microvessels (Fig. 1). However, contraction diminished and the microvessels relaxed after the BSCF was washed out, showing that the effects of BSCF were reversible (Fig. 1A and $\mathrm{B}$ ). The mean contraction percentage of retinal microvessels following BCSF exposure was significantly lower compared with prior to BCSF exposure $(\mathrm{P}<0.01$; Fig. 1C).

BCSF induced contraction of pericyte-populated collagen gel and increased $\alpha$-SMA expression. Cultured pericytes were identified using $\alpha$-SMA as a marker and morphological images after the 3rd passage (Fig. 2A and B). With the addition of pericytes, collagen gels contracted to $63 \%$ of their initial diameter at $24 \mathrm{~h}(\mathrm{P}<0.05$; Fig. $2 \mathrm{C}-\mathrm{E})$. The addition of $\mathrm{BCSF}$ significantly accelerated pericyte-populated collagen gel contraction at $24 \mathrm{~h}$ to $36 \%(\mathrm{P}<0.05$; Fig. $2 \mathrm{C}-\mathrm{E})$. The increased contraction induced by BCSF exhibited a time-dependent trend over $24 \mathrm{~h}$ in groups treated with saline and BCSF (Fig. 2D). The BCSF-treated pericytes group and saline-treated control pericyte group from the collagen gel expressed different levels of $\alpha$-SMA. Western blots identified that the expression of $\alpha$-SMA was significantly upregulated over a 2-day period of incubation with BCSF ( $\mathrm{P}<0.05$, Fig. $2 \mathrm{~F})$.

BCSF induced $\left[\mathrm{Ca}^{2+}\right]_{i}$ elevation in cultured retinal pericytes. Microfluorimetry analysis identified that pericyte exposure 
to $\mathrm{BCSF}$ produced a peak $\left[\mathrm{Ca}^{2+}\right]_{\mathrm{i}}$ response, followed by a sustained plateau phase in the presence of extracellular $\mathrm{Ca}^{2+}$ (Fig. 3A and B). BCSF exposure in the absence of extracellular $\mathrm{Ca}^{2+}$ dramatically decreased the peak $\left[\mathrm{Ca}^{2+}\right]_{i}$ response, producing only a small transient peak without a plateau phase (Fig. 3C).

\section{Discussion}

In the present study, it was identified that BCSF exposure induced retinal microvessel contraction and that this contraction was resolved by BCSF wash-out. In addition, BCSF exposure accelerated retinal pericyte-populated collagen gel contraction and increased the expression of $\alpha$-SMA in a time-dependent manner. Furthermore, BCSF induced a calcium influx in cultured retinal pericytes.

Numerous studies of SAH in the past decades have focused on vasospasm in the primary arteries $(4,17,18)$. It was widely accepted that the ischemia that prognosticates poor outcome was caused by cerebral vasospasm (18). However, this classical theory of SAH-induced vasospasm has been fiercely challenged by a clinical trial of clazosentan, which, although reversing angiographic vasospasm, failed to improve patient outcome (19). Previous clinical studies have showed that numerous patients develop angiographic vasospasm following aneurysmal SAH, but only a small number will go on to develop cerebral ischemia and infarction (18,20-22). Angiographic vasospasm does not always correlate with poor clinical outcome $(18,19)$, this may be attributable to the changes in microcirculation following SAH $(6,23,24)$.

Previous studies have demonstrated that the cerebral microvasculature is significantly affected by $\mathrm{SAH}(6,7,25)$. Uhl et al (26) reported, for the first time, direct visualization of cerebral microcirculatory changes in patients following SAH with orthogonal polarization spectral imaging. Similar observations were described by Friedrich et al (27). These studies showed that $\mathrm{SAH}$ is associated with a micro-vasospasm, primarily affecting arterioles, with a reduction of diameter in pial vessels and an overall decrease in microvessel density. It is postulated that the increased intracranial pressure following SAH leads to compression in cerebral microvessels, resulting in a reduction in microvessel density (28). However, due to technical limitations, these studies showed no direct evidence of microvessel dysfunction following SAH.

The present study identified that SAH may be associated with increased contractility of microvessels, as indicated by enhanced vasoconstriction in response to BCSF. As microvessels lack smooth muscle, blood flow is frequently assumed to be regulated by precapillary arterioles. However, the majority (65\%) of adrenergic innervation of central nervous system (CNS) blood vessels terminates near microvessels rather than arterioles, and in the muscle and brain a dilatory signal propagates from vessels near metabolically active cells to precapillary arterioles, suggesting that blood flow control is initiated in microvessels $(4,5,7)$. Pericytes in CNS microvessels contain contractile proteins and can initiate such signaling $(29,30)$. In the present study it was observed that pericytes can control microvessel diameter in whole-mount retinal microvasculature.
A
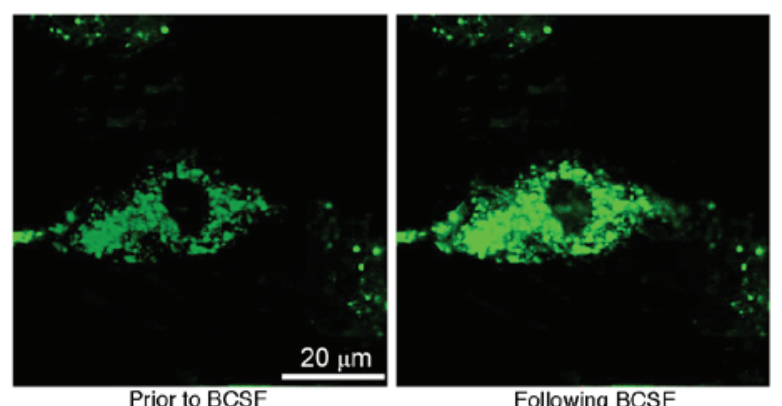

B

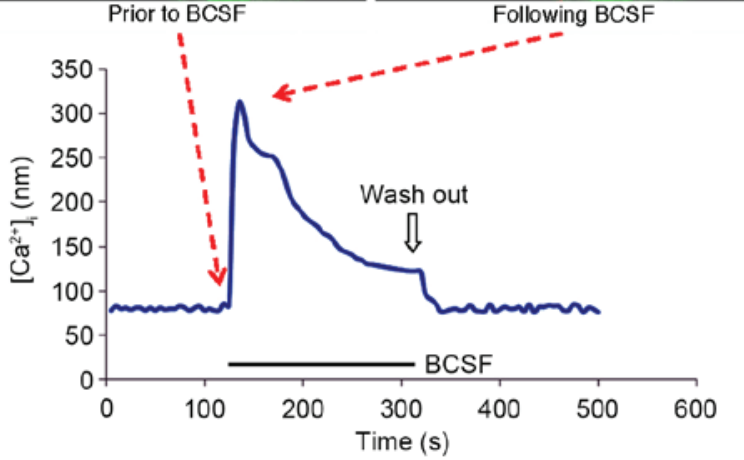

$\mathrm{C}$

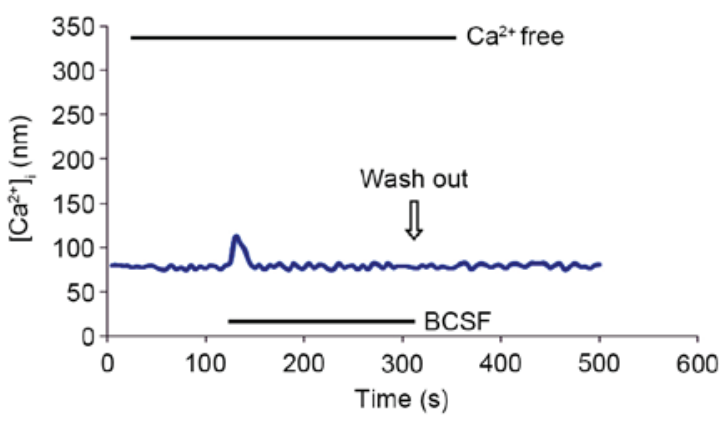

Figure 3. BCSF-induced $\left[\mathrm{Ca}^{2+}\right]_{\mathrm{i}}$ elevation in retinal pericytes. (A) Representative images of $\left[\mathrm{Ca}^{2+}\right]_{i}$ prior to and following BCSF incubation. (B) In the presence of extracellular $\mathrm{Ca}^{2+} \mathrm{BCSF}$ produced a biphasic $\left[\mathrm{Ca}^{2+}\right]_{\text {i }}$ response, with a transient peak and a prolonged plateau phase. The effect of BCSF was reversible by wash out. (C) In the absence of extracellular $\mathrm{Ca}^{2+}$ BCSF induced a small peak $\left[\mathrm{Ca}^{2+}\right]_{i}$ response without a plateau phase. $n=5$ experimental repeats. BCSF, blood-filled cerebrospinal fluid.

It has often been postulated that pericytes are contractile cells and contribute to the regulation of blood flow at the microvascular level $(29,30)$. The BCSF-induced microvessel observed in the present study may be attributable to the release of a vasoactive agent from underlying glia and neurons. In order to exclude this cause, the effect of BCSF on cultured pericytes was tested with a gel contraction assay. This identified that BCSF accelerated pericyte-populated collagen gel contraction over $24 \mathrm{~h}$. This confirms that BCSF affects microvessel function through enhancing pericyte contraction. Future studies into the underlying mechanisms and role of pericyte-containing microvessel disturbances in the pathophysiology of delayed cerebral ischemia should be explored.

In conclusion, the present study demonstrates an increased contractility of the pericytes and reduced diameter of microvessels in the presence of BCSF in vitro. These findings indicate that pericyte contraction and microvascular dysfunction is induced following $\mathrm{SAH}$, which may lead to an increased susceptibility to SAH-induced ischemia. 


\section{Acknowledgements}

The present study was supported by the National Natural Science Foundation of China (grant no. 30801186, 81501002 and 81220108009) and the National Basic Research Program of China (program 973, grant no. 2014CB541600).

\section{References}

1. Woitzik J, Dreier JP, Hecht N, Fiss I, Sandow N, Major S, Winkler M, Dahlem YA, Manville J, Diepers M, et al: Delayed cerebral ischemia and spreading depolarization in absence of angiographic vasospasm after subarachnoid hemorrhage. J Cereb Blood Flow Metab 32: 203-212, 2012.

2. Macdonald RL: Delayed neurological deterioration after subarachnoid haemorrhage. Nat Rev Neurol 10: 44-58, 2014.

3. Meyers PM and Connolly ES Jr: Stroke: Disappointing results for clazosentan in CONSCIOUS-2. Nat Rev Neurol 7: 660-661, 2011.

4. Chen S, Feng H, Sherchan P, Klebe D, Zhao G, Sun X, Zhang J, Tang $\mathrm{J}$ and Zhang JH: Controversies and evolving new mechanisms in subarachnoid hemorrhage. Prog Neurobiol 115: 64-91, 2014.

5. Chen Y, Li Q, Tang J, Feng H and Zhang JH: The evolving roles of pericyte in early brain injury after subarachnoid hemorrhage. Brain Res 1623: 110-122, 2015.

6. Tso MK and Macdonald RL: Subarachnoid hemorrhage: A review of experimental studies on the microcirculation and the neurovascular unit. Transl Stroke Res 5: 174-189, 2014.

7. Ostergaard L, Aamand R, Karabegovic S, Tietze A, Blicher JU, Mikkelsen IK, Iversen NK, Secher N, Engedal TS, Anzabi M, et al: The role of the microcirculation in delayed cerebral ischemia and chronic degenerative changes after subarachnoid hemorrhage. J Cereb Blood Flow Metab 33: 1825-1837, 2013.

8. Institute of Laboratory Animal Resources (US). Committee on Care, Use of Laboratory Animals and National Institutes of Health (US). Division of Research Resources: Guide for the care and use of laboratory animals. 8th edition. National Academies Press, Washington, DC, 2011.

9. Foley PL, Takenaka K, Kassell NF and Lee KS: Cytotoxic effects of bloody cerebrospinal fluid on cerebral endothelial cells in culture. J Neurosurg 81: 87-92, 1994.

10. Sun W, Deng Q, Levick WR and He S: ON direction-selective ganglion cells in the mouse retina. J Physiol 576: 197-202, 2006.

11. Kawamura H, Kobayashi M, Li Q, Yamanishi S, Katsumura K, Minami M, Wu DM and Puro DG: Effects of angiotensin II on the pericyte-containing microvasculature of the rat retina. J Physiol 561: 671-683, 2004.

12. Wu DM, Kawamura H, Sakagami K, Kobayashi M and Puro DG: Cholinergic regulation of pericyte-containing retinal microvessels. Am J Physiol Heart Circ Physiol 284: H2083-H2090, 2003.

13. Liu G, Meng C, Pan M, Chen M, Deng R, Lin L, Zhao L and Liu X: Isolation, purification, and cultivation of primary retinal microvascular pericytes: A novel model using rats. Microcirculation 21: 478-489, 2014.
14. Oishi $\mathrm{K}$, Kamiyashiki $\mathrm{T}$ and Ito $\mathrm{Y}$ : Isometric contraction of microvascular pericytes from mouse brain parenchyma. Microvasc Res 73: 20-28, 2007.

15. Chen Y, Zhang Y, Tang J, Liu F, Hu Q, Luo C, Tang J, Feng H and Zhang JH: Norrin protected blood-brain barrier via frizzled- $4 / \beta$-catenin pathway after subarachnoid hemorrhage in rats. Stroke 46: 529-536, 2015.

16. Wurm A, Pannicke T and Reichenbach A: Ca2+ microfluorimetry in retinal Müller glial cells. Methods Mol Biol 935: 257-270, 2013.

17. Caner B, Hou J, Altay O, Fujii M and Zhang JH: Transition of research focus from vasospasm to early brain injury after subarachnoid hemorrhage. J Neurochem 123 (Suppl 2): S12-S21, 2012.

18. Macdonald RL, Pluta RM and Zhang JH: Cerebral vasospasm after subarachnoid hemorrhage: The emerging revolution. Nat Clin Pract Neurol 3: 256-263, 2007.

19. Wong GK and Poon WS: Clazosentan for patients with subarachnoid haemorrhage: Lessons learned. Lancet Neurol 10: 871-872, 2011.

20. Cossu G, Messerer M, Oddo M and Daniel RT: To look beyond vasospasm in aneurysmal subarachnoid haemorrhage. Biomed Res Int 2014: 628597, 2014.

21. Hou J and Zhang JH: Does prevention of vasospasm in subarachnoid hemorrhage improve clinical outcome? No. Stroke 44 (6 Suppl 1): S34-S36, 2013.

22. Hansen-Schwartz J, Vajkoczy P, Macdonald RL, Pluta RM and Zhang JH: Cerebral vasospasm: Looking beyond vasoconstriction. Trends Pharmacol Sci 28: 252-256, 2007.

23. Terpolilli NA, Brem C, Bühler D and Plesnila N: Are we barking up the wrong vessels? Cerebral microcirculation after subarachnoid hemorrhage. Stroke 46: 3014-3019, 2015.

24. Naraoka M, Matsuda N, Shimamura N, Asano K and Ohkuma H: The role of arterioles and the microcirculation in the development of vasospasm after aneurysmal SAH. Biomed Res Int 2014: 253746, 2014.

25. Chai WN, Sun XC, Lv FJ, Wan B and Jiang L: Clinical study of changes of cerebral microcirculation in cerebral vasospasm after SAH. Acta Neurochir Suppl 110: 225-228, 2011.

26. Uhl E, Lehmberg J, Steiger HJ and Messmer K: Intraoperative detection of early microvasospasm in patients with subarachnoid hemorrhage by using orthogonal polarization spectral imaging. Neurosurgery 52: 1307-1317, 2003.

27. Friedrich B, Müller F, Feiler S, Schöller K and Plesnila N: Experimental subarachnoid hemorrhage causes early and long-lasting microarterial constriction and microthrombosis: An in-vivo microscopy study. J Cereb Blood Flow Metab 32: 447-455, 2012.

28. Chen S, Chen Y, Xu L, Matei N, Tang J, Feng H and Zhang JH: Venous system in acute brain injury: Mechanisms of pathophysiological change and function. Exp Neurol 272: 4-10, 2015.

29. Peppiatt CM, Howarth C, Mobbs P and Attwell D: Bidirectional control of CNS capillary diameter by pericytes. Nature 443: 700-704, 2006

30. Hall CN, Reynell C, Gesslein B, Hamilton NB, Mishra A, Sutherland BA, O'Farrell FM, Buchan AM, Lauritzen M and Attwell D: Capillary pericytes regulate cerebral blood flow in health and disease. Nature 508: 55-60, 2014. 